\title{
Patient-provider communication and reproductive health among HIV-positive women in Rio de Janeiro, Brazil
}

\author{
Monica Malta ${ }^{\mathrm{a}, *}$, Catherine S. Todd ${ }^{\mathrm{b}}$, Mark A. Stibich ${ }^{\mathrm{c}}$, Thais Garcia ${ }^{\mathrm{a}}$, Diego Pacheco ${ }^{\mathrm{a}}$, Francisco I. Bastos ${ }^{\mathrm{a}}$ \\ a Oswaldo Cruz Foundation, Rio de Janeiro, Brazil \\ ${ }^{\mathrm{b}}$ Department of Obstetrics \&' Gynecology, Columbia University, New York, NY, USA \\ ${ }^{\mathrm{c}}$ Lacuna Consulting, Houston, TX, USA
}

\section{A R T I C L E I N F O}

Article history:

Received 1 March 2010

Received in revised form 14 September 2010

Accepted 15 September 2010

\section{Keywords:}

HIV

AIDS

Reproductive health

Women

Brazil

\begin{abstract}
A B S T R A C T
Objective: To qualitatively assess the influence of patient-provider communication on contraceptive choice among HIV-positive women in the context of universal antiretroviral therapy (ART) access Methods: Focus group discussions (FGD; $n=3$ ), in-depth (IDI; $n=15$ ) and freelist interviews (FLI; $n=36$ ) were conducted with HIV-positive women aged 18-40 years recruited from public health units in Rio de Janeiro/Brazil.

Results: Of 70 participants, 49 used ART and the median time since HIV diagnosis was 6 years (range: 1 18). The majority of participants (71.4\%) reported some degree of dissatisfaction with their health providers (usually lack of open dialogue) and a few reported experiences of stigma/prejudice during appointments. Intra, interpersonal and social factors modulated behaviors and reproductive health decisions, and those issues were rarely addressed by providers during HIV clinical care.

Conclusion: Despite dramatic increases in survival and life quality after universal ART implementation in Brazil, reproductive health issues are neglected by multiple cadres of HIV health providers. Communication on reproductive health issues remains fragmented and potentially contradictory, compromising care in these settings.

Practice implications: Adequate provider training to address reproductive health-related issues in a comprehensive, culturally sensitive manner and improved integration of HIV and reproductive health care are urgently needed in this setting.
\end{abstract}

(c) 2010 Elsevier Ireland Ltd. All rights reserved.

\section{Introduction}

An estimated 33 million people are infected with HIV globally, and half are women [1]. Women of reproductive age are disproportionately affected by the HIV/AIDS epidemic; consequently, family planning and HIV-related interventions intersect in a number of crucial ways. Despite the established connections between the fields, bridging of these two areas occurs with varying degrees of effectiveness in clinical practice. In settings where family planning interventions have been underutilized in HIV prevention, care, and treatment programs, rates of unintended pregnancy remain high among women living with HIV [2-4].

Brazil was the first middle-income country to provide full access to antiretroviral therapy (ART), laboratory monitoring, and clinical

\footnotetext{
* Corresponding author at: Oswaldo Cruz Foundation, Social Science Department, National School of Public Health (DCS/ENSP), Rua Leopoldo Bulhoes, 1480, room 905, Manguinhos, Rio de Janeiro, RJ 21041-210, Brazil. Tel.: +55 212598 2715; fax: +552125982779.

E-mail addresses: malta@ensp.fiocruz.br, mmalta@jhsph.edu (M. Malta).
}

care at no cost to any eligible patient at the point of health care delivery. This national program, initiated in 1996, has progressively expanded, with the decentralization of ART dispensing units and a comprehensive network of laboratory monitoring, including regular CD4 counts, viral load measures and genotyping [5]. A direct result of this initiative is a significant increase in survival following diagnosis, particularly for those diagnosed with HIV after 1996. This scenario presents new challenges to patients, health providers, and policy makers, since women of reproductive age living with HIV/AIDS report the desire to have children and require effective reproductive health services tailored to their specific needs $[3,6]$.

Women living with HIV/AIDS can improve the quality of their reproductive health care by actively communicating with physicians, nurses, psychologists and other providers. A few published papers suggest that when patients fully disclose their concerns, expectations, and preferences, providers trained in enhanced communication methods might be able to assess their problems more accurately and offer better advice $[7,8]$. According to Newbold and Willinsky, when patients receive reproductive information that is culturally adequate and accessible for their educational levels, they are more likely to make better informed 
decisions, feel more control over those decisions, and are more committed to implementing those decisions [9].

In Brazil, HIV-positive women have identified a great desire for future fertility, lack of proper access to reproductive health care, and use of less effective contraceptive methods (e.g. withdrawal) [10-13]. Participants also reported lack of counseling towards reproductive health and contraception during their monthly appointments [10-13]. One study conducted with Brazilian HIVpositive women of reproductive age identified a strong desire for children, even among women with at least one child already [14]. However, these issues receive little attention in patient care as the authors found uneven access to sexual and reproductive health counseling. Interviewed physicians reported never discussing condom use, contraceptive methods, or reproductive health issues with their HIV-positive patients [14].

A large study conducted with 1068 women living with HIV/ AIDS from São Paulo identified that 15\% were considering becoming pregnant and, among those with long-term partnerships, 63\% used condoms in all sexual relations, which was three times the national average [15]. Knowledge about HIV vertical transmission was lower than expected and participants complained of limited space and receptiveness of health professionals for discussing sexuality, particularly related to future fertility, leading the authors to conclude that access to reproductive health counseling was inadequate for HIV-positive patients [15].

The purpose of this qualitative study was to evaluate what values and preferences HIV-positive women have regarding communication with their providers about reproductive health, specifically decisions surrounding fertility timing and choice. Although a few quantitative studies are available about this important topic, there is a lack of qualitative investigation that might lead to an in-depth understanding of the possibilities (and gaps) in reproductive health dialogue among this highly vulnerable set of patients. Further, qualitative studies provide further insight into the context of reproductive health decision-making and the relative role of the provider within that process. The results may increase our knowledge and guide culturally informed counseling efforts better tailored to the needs, personal and social values of women living with HIV/AIDS.

\section{Methods}

The information for this manuscript emerged from a larger, three-site qualitative study whose primary aim was to explore the perceptions of HIV-positive women towards contraceptive choice and discontinuation. The parent study was conducted in Soweto, South Africa; Kericho, Kenya, and Rio de Janeiro, Brazil. However, in the Brazil site, there was frequent spontaneous commentary regarding the perceived quality of reproductive health care from a variety of providers and on barriers to discussing reproductive health issues with HIV-care providers that prompted this separate analysis.

\subsection{Participants}

We conducted this qualitative assessment among HIV-positive women aged 18-40 years able to provide informed consent and receiving HIV care at public health facilities in Rio de Janeiro, Brazil. Approvals were received from the institutional review boards of National School of Public Health (CEP/ENSP) in Brazil, the University of California, San Diego, and Columbia University.

\subsection{Data collection}

Potential participants were identified during clinic visits, screened for inclusion criteria, and taken to a private room for study information and informed consent. Participants completed a standardized screener about HIV, ART, obstetric history, and contraceptive utilization and then completed the appointed interview conducted by trained female study staff.

All participants were recruited in three public HIV-care facilities located in downtown, Rio de Janeiro. Those recruitment sites were chosen because they represent the standard of care available in Rio de Janeiro, outside reference centers. The study was initially presented to all patients in the waiting room, and latter on, those who demonstrated wiliness to participate were individually interviewed after their clinical appointments. Focal group participants were recruited by their primary HIV-care providers. Eligible participants were women HIV-positive, aged 18-40, able to understand the consent form and under HIV-treatment in selected public outpatient unit.

\subsection{Interview methods and content}

The following qualitative methods were adopted: three focus groups discussions (FGD), 15 in-depth interviews (IDI) and 36 freelist interviews (FLI) with participants. Freelist participants were purposively selected based on three age ( $<23$ years; $23-30$ years, $>30$ years $)$ and parity $(0,1-2,>2)$ strata creating nine possible groups, ensuring even distribution of potential confounders. FGD and IDI participants were selected by age strata only. We elected to perform a qualitative study to better understand the relative impact of different individual, structural, and environmental factors on reproductive health decision-making among HIV-positive women, which includes interactions with health care providers.

FGD were conducted to gain insight into the main themes for each topic of interest. A field guide was piloted with one group for refinement and assessment of question understanding. With the finalized field guide, three separate hour-long FGD were conducted by a moderator with a session recorder present to capture nonverbal communication and areas of group agreement/disagreement. FGD questions addressed women's attitudes toward fertility, contraception, and the impact of HIV and partnership on these attitudes. Interaction with medical providers was probed when it spontaneously arose within these group discussions. Investigators reviewed the resultant transcripts to develop IDI questions.

IDI were conducted among 15 women to further explore emergent FGD themes. IDI were approximately 45 min each. The IDI guide utilized the "grand tour" approach in which the participant is asked a broad question to encourage her to speak openly about the topic. The "grand tour" question bounds the interview while still encouraging detailed responses from the participant [16]. We employed the grand tour question twice to assess beliefs about contraception: "Tell me everything you know about birth control" and "Tell me everything you think about what a perfect prevention method should be like." A series of probe questions were placed after each grand tour question in the IDI guide to remind the interviewer of specific issues to be addressed if not spontaneously raised by the participant; probes relevant to the patient-provider relationship included method availability and barriers to access. Other IDI questions invited participants to share their perceptions on the meaning, significance, and feelings around contraception and amenorrhea, including how HIV status affects contraceptive choice. Participant's perception on fertility and contraception are reported elsewhere; herein we will address specifically issues around patient-provider communication [17]. Responses to queries about dialogue and relationship with care providers are included in this analysis.

Freelist methodology was developed to sample terms contained within a given category (e.g. cultural domain), without imposing investigator assumptions on respondents $[18,19]$. This technique, 
which contains elements of both qualitative and quantitative methods, allows for qualitative data collection while providing a means to rapidly analyze data and provide timely information. Participants create a list of items contained within a category with the interviewer repeating the list prompts until the respondent can think of no additional items. In general, 30 participants will fully elucidate a cultural domain [20].

Freelist statements were developed from input from the FGD and IDI. FL statements were refined through an iterative process that began with the local study staff collaborating with the full research team to develop multiple statements that met the research objectives while conforming to the local understanding of the cultural domain. Once constructed, the statements were pretested to assure content validity of the statements being used $[16,21]$. Each participant was asked individually multiple freelist questions addressing preferred contraceptive methods, issues related to pregnancy desire among HIV-positive women and communication with health professionals about pregnancy desire and contraceptive methods.

\subsection{Data analysis}

Transcribed IDI and FGD were read to discern participants' views and behaviors regarding reproductive health and patient-provider communication. Descriptive notes were written in the margins to facilitate coding by two of the authors (MM and TG), these were compared, contrasted and merged to create a single coding scheme. Text with similar codes was examined and compared across interviews, leading to preliminary themes. Codes were generated inductively, using 'open coding' methodology [22]. All coded data segments reviewed line-by-line to create sub-codes. FGD and IDI transcripts were analyzed using principles of grounded theory [22]. Selected quotes were included to illustrate major research findings reported by the interviewees. The selection of quotes aimed at covering all expressed viewpoints while avoiding redundancy.

For FLI, descriptive statistics were generated for demographic and reproductive health characteristics. Primary freelist analysis was conducted in two phases [18]. The first phase was language standardization across lists. Freelists were then analyzed using ANTHROPAC version 4.98 to examine the frequencies and saliency of each item [23]. Saliency was measured using Smith's S - a frequency count weighted inversely by an item's rank in each list [24]. Age, parity, and ART status were compared to demographic variables and select freelist mentions with Chi-square testing to gain additional insight on how patient-provider communication impacted contraceptive and fertility decision-making. As we collected data stratified by age group and parity, any differences between the specified strata are identified by theme and presented in Section 3.

\section{Results}

\subsection{Demographics}

We conducted 3 FGD (19 participants), 15 IDI, and 36 FLI with 70 total participants; demographic and reproductive health characteristics are summarized in Table 1. Participants were young women (mean age 28.3 , range $18-40$ ), with $70.0 \%$ using ART and $47.1 \%$ unmarried. The median time since HIV diagnosis was 6 years (range: 1-18). Fourteen participants reported a great desire to have children and 12 were pregnant at the time of interview ( $20.0 \%$ and $17.1 \%$, respectively).

\subsection{Thematic overview}

Overall, the transcripts of interviews were rich and participants seemed willing to speak freely and in-depth. Participants'
Table 1

Demographic and reproductive health characteristics of HIV-infected women interviewed in Rio de Janeiro, Brazil $(n=70)$.

\begin{tabular}{ll}
\hline Characteristic & $N(\%)$ \\
\hline Age (mean, range) & 28.3 years $(18-40)$ \\
On ART & $49.0(70 \%)$ \\
Marital status & $33(47.1 \%)$ \\
Single & $26(37.1 \%)$ \\
Married & $6(8.6 \%)$ \\
Divorced & $5(7.1 \%)$ \\
Widowed & \\
Pregnancies (average per participant) & 1.5 \\
Live births & 1.3 \\
Miscarriages & 0 \\
Abortions & \\
Last contraceptive method used & $28(40.0 \%)$ \\
Condom & $16(22.9 \%)$ \\
Condom and pill & $14(20.0 \%)$ \\
Tubal ligation & $4(5.7 \%)$ \\
Pill & $1(1.4 \%)$ \\
Abstinence & $1(1.4 \%)$ \\
Condom and tubal ligation & $1(1.4 \%)$ \\
Condom and Depo-Provera & $1(1.4 \%)$ \\
Female condom & $1(1.4 \%)$ \\
Perlutan injection & $1(1.4 \%)$ \\
Pill and withdrawal & $1(1.4 \%)$ \\
Withdrawal & $1(1.4 \%)$ \\
None &
\end{tabular}

perception of their health care providers was organized on the following three major themes: overall satisfaction, negative and positive experiences of counseling. Those themes include experiences related to communication and support with health care providers, level of satisfaction, and quality of discussion around fertility aspects of reproductive health.

\subsection{Overall satisfaction}

Our findings point to a low level of satisfaction with health providers in general - among 70 participants, 50 reported some degree of dissatisfaction with their HIV-care provider, attending nurses, or social workers (71.4\%). Key problems included less than optimal patient-provider dialogue, lack of empathy, and experiences of stigma/prejudice during appointments.

Both nurses were really mean to me when they found out I was trying to get pregnant, they even told me to get an abortion [illegal and highly stigmatized in Brazil]. [37 years old, HIV-positive since 1997, FLI]

Asymmetrical relationships between primary HIV-care providers and patients were very common, especially when discussing and deciding whether or not to conduct a tubal ligation as identified in a few in-depth interviews:

I did it because when I had my second daughter, the doctors told me: You should do a ligature, so you will not risk yourself anymore.

You already have two daughters. So, they told me to do the ligature [tubal sterilization]. I felt a deep regret. Very deep! [36 years old, HIV-positive since 1999, IDI]

Participant: I was mad with my doctor because he didn't want to do this [to perform the tubal sterilization] on me.

Interviewer: Did you want it?

Participant: Oh, yes, but he said women are using this method and don't use condoms anymore, understand? He said many, many things I did not agree but. . I don't have money to do it on my own [in a private clinic], but I would like to do it. [33 years old, HIV-positive since 2002, IDI] 
Though the positions of those providers were diametrically opposite, both reflect a paternalistic approach where the common assumption is that the patient could not be trusted to care for herself. Surgical sterilization was the method that appeared to incite this level of discord, likely reflective of its permanence. Other patients reported that they had a better and more open relationship with attending nurses and social workers rather than with their HIV-care provider:

Like, I love this nurse, he is so funny. . I love talking to him, and he keeps teasing me all the time, sometimes he gives me condoms with strawberry flavor and stuff like that. He is cool, like, he seems more like a friend of mine than my doctor, because my doc is just. . too serious, you know? So I don't feel like talking about sex with him. . . [22 years old, HIV-positive since 2008, FLI]

A few participants also reported being satisfied with their health care provider, particularly when the outcome was successful:

"Everybody thinks about having a family, but I kept thinking: I can't be a mother, I can't be a mother anymore! But I've started to study about all such things (...). I got informed myself that there were people that could get pregnant even in my condition... that could do a trustful treatment before the pregnancy. One should take all the necessary treatment during pregnancy. So I started to get very interested about this subject. Then now, last year, I realized I could have it [be pregnant] and I saw a doctor, who counseled me... And we did it, see? [got pregnant] Did it just right. I'm infected for a lot of years now. So is he [her current husband]. Then I said: - We're gonna have a babe now. So far the exam [viral load] didn't detect anything, thanks God. He [the baby] didn't even get a flu, so far. He is very healthy. Nothing, nothing, nothing. [38 years old, HIV-positive since 1999, IDI]

\subsection{Negative experiences with health providers}

The majority of participants complained about a lack of support and communication during appointments with their HIV-care provider $(N=63,9 \%)$. Participants frequently stated that they had lingering doubts or concerns that they did not fully articulate to their providers during the consultation prior to their interview. The primary reason given for not raising these concerns with providers was the lack of openness to discuss their personal problems, illustrated in the following quotations:

Like, they [health care providers] keep telling me to always use condoms, but they don't want to hear my stories, they don't want to really understand whatever is going on with me, why I'm always drinking and hanging out with crazy guys... They just want to prescribe something, like condoms, without knowing the real truth. They can't handle it, and I keep it to myself, and share only with those who seem to understand and at least to listen to me... They always keep the same script. Are you using condoms? And I: Sure, always. Then they give me more condoms, and that's it. No one asks me if I like to use it, if I need to talk about my sexual life, or anything. So, it's a truly empty talk from both sides. They don't want to hear me, and I pretend to keep up with whatever they want me to do. It's a big joke, that's the truth. [25 years old, HIV-positive since 2005, IDI]

Participants also mentioned a lack of understanding on the part of the health care providers of the full context surrounding condom use:

She is not a very friendly doctor... I feel afraid of her sometimes, you know? She got really upset when I got pregnant with my last daughter, I felt really bad. But couldn't tell her that it wasn't my fault, I just couldn't say no to my husband. He looks after me, feeds me and the kids, I just couldn't deny him. . . My doctor always tells me to use condoms, always give me condoms, but she never asked me if I really use it, or if my husband accepts to use it. It's like something automatic, you know? She tells me to keep using condoms, give me three or six condoms every appointment, but never asks what I think about it. I take the condoms, put in my purse to avoid any argument, and later on I give it to a friend of mine... [25 years, HIV-positive since 2006, FLI]

Participants also mentioned multiple challenges concerning discussing reproductive health issues with health care providers. These challenges included the avoidance (by the health care worker) of the subject of sex, judgmental tones, and failure on the part of the health care worker to listen to the participant. There was a focus on education about condoms from the health care providers, without the health care worker first assessing the context in which condoms would be used or the level of knowledge of the participant about condoms.

Interviewer: Now could you tell me everything your doctor has told you, since your diagnosis, about birth control and pregnancy?

Participant: Condoms, condoms and condoms. It's like a brainwash, they keep giving you condoms every appointment, seems like you're an evil person that just wants to spread diseases, and it's not true. [40 years old, HIV-positive since 1998, FLI]

Some participants indicated that any mention of reproductive health was limited to a one-way public service type of statement at the end of an appointment, and this environment was not perceived as conducive to further discussion of personal reproductive issues.

You know, they just can't handle the conversation about sex, so they usually tell you by the end of your appointment to get a few condoms with the nurse. They absolutely avoid the conversation, and that makes me wonder... What kind of doctor are they? [19 years old, HIV-positive since 2006, FLI]

Participants also mentioned that they felt ashamed when they could not follow advice (such as "use condoms") due to contextual circumstances. Sometimes, this asymmetrical relationship leaded to serious consequences, as follows:

He [physician] told me about condoms, and I felt ashamed of telling him that my husband didn't accept it. One day, when I came home with condoms in the purse that the doctor gave me here, my husband beat me really hard. He thought that I was with another man. He was drunk. . . He even broke my arm, it was terrible. My kids saw it, but they were too afraid to ask for help. I never told my doctor about it, and every appointment when he gave me the condoms, I accept it and throw them away in the first garbage... [35 years, HIV-positive since 1999, FLI]

Overall, HIV-positive women had poor access to the necessary dialogue with health care providers in general, demonstrating asymmetries in dialogue and relationship between patientprovider, an important limitation towards adequate treatment and care of this population.

\subsection{Positive experiences with health providers}

A few participants reported two aspects of support from health care providers that were positive: clarifying misconceptions about HIV/AIDS and assisting with family. These mentions were in the minority $(N=10,14.3 \%)$, but are important to highlight, as they represent areas of successful interaction. 
Yes... I was afraid he [partner] would never touch me again... [after the HIV-diagnosis] these things... I was scared because I didn't know about this disease, but then I talked with a psychologist and a doctor and the things became clearer in my mind. [33 years, HIV-positive since 1999, IDI]

The challenges of dealing with disclosure of HIV were also mentioned by a few participants. Health care providers need to receive appropriate training on this highly sensitive issue, since they are frequently faced with a two-side story: the perceived high risk to the third party on one hand and the ethical obligation to "respect persons" in terms of privacy and confidentiality on the other side. This participant reports an experience where health professionals' dialogue and psychological support was decisive to allow her husband disclosure of his HIV status.

So when he [her husband] figured out he was infected... see? He didn't want to tell me. It was very hard for him to tell. But then he was convinced [by the health professionals in charge of his treatment] to tell me. He was being treated behind my back. In the hospital he was convinced to tell me the truth. So he told me, I came, I did the test, and then we realized that me and my daughter were also infected. [33 years, HIV positive since 1998, IDI]

A small percentage of participants also reported access to an open-minded and receptive environment to address their concerns and doubts about reproductive choices, in a setting providing open dialogue with their HIV-care providers $(N=7,10 \%)$. Those patients clearly demonstrated the positive impact of this adequate communication on their daily lives:

We [patient and provider] talk about condoms, and we talk a lot about the possibility of having a broken condom, because my husband is negative. Before we started having sex, he came here for an appointment, to listen and ask all his questions without me. I thought he will feel more comfortable this way, and I was right. Later on, my doctor talked a lot with me, and explained to me a lot of things about my infection, about how my viral load was low, and how condoms were effective to protect him (partner). I was really worried, but everything is okay now. We live a normal life, got married, but we never had sex without a condom, and he is okay with that. [39 years, HIV-positive since 1997, FLI]

Unfortunately those successful experiences were not widespread within participants, highlighting the need to develop better interventions targeting HIV-positive women.

\subsection{Results summary}

Overall, the data presented rich quotations concerning patientprovider interactions and relationships. Participants indicated that contextual factors, primarily their relationship with their own sexual partner, were a primary driver behind condom use and that these factors were not acknowledged, discussed or understood by their health care provider. Additionally, participants provided quotations concerning the overall level of support, the quality of communication and other insight into the patient-provider interaction in this context.

\section{Discussion and conclusion}

\subsection{Discussion}

The study findings indicate that there are potentially many missed or ineffectively utilized opportunities to address contraceptive use, planned pregnancy, and protected sex among people living with HIV/AIDS (PLWHA) in ongoing HIV clinical care consultations. Many participants expressed dissatisfaction with their providers and/or health professionals in general. Those findings are similar to previous studies conducted among physicians and with both patient and providers in Rio de Janeiro $[25,26]$.

While a few providers in this study engaged in some level of communication regarding reproductive health with their patients, the form of communication was often closed-ended and perfunctory. Most providers did not communicate at all with their patients about sexual behavior, fertility intentions, or contraception. The few exceptions to this had a great positive impact on quality of life of both the female patient and her male partner, reinforcing the need to include this discussion topic in every appointment, either with the primary provider or with other health professionals with more time and/or more skills to conduct this discussion. Rather than offering condoms at the end of an appointment, or distributing condoms without any discussion about the patient's interest and ability to negotiate their use, providers could engage their patients in an open discussion about protected sex. Additional staff (e.g. nurses, social workers, psychologists) could also initiate discussions about gender issues and decision-making about condom use, trying to improve patient empowerment in their stable and occasional relationships.

HIV-positive women of reproductive age need contraception to avoid unintended pregnancy, both to preserve their own health and to reduce the risk of transmitting HIV to an infant [27]. Although some providers seemed more open to discussing reproductive choices than safer sex issues with their patients, a few participants faced stigma, resistance, and discrimination from their providers when they decided to get pregnant. The lack of proper counseling, coupled with a patient's desired fertility, suggest that even in the Brazilian context of free universal access to ART, patients lack appropriate reproductive health and family planning services to ensure fully informed decisions about when and whether to have children. This is not a new problem, as has been discussed by several authors [2,3]. The self-reported current contraceptive method of most participants was male condoms, though many women disclosed barriers to use and relayed an inability to explore techniques to overcome these barriers with their physician or other medical provider. Empowerment to affect condom use and partner support for contraceptive use could be increased if better counseling and psychosocial support were accessible during or in combination with HIV/AIDS treatment.

There was discussion related to other contraceptive methods besides condoms. Though some women utilized oral contraceptives, most comments centered on female surgical sterilization, the most common contraceptive method used in Brazil [28]. An estimated $44 \%$ of all Brazilian women aged between 14 and 55 had been permanently sterilized [29]. Sterilization is required by law to have a waiting period with certain age and partnership criteria. However, much latitude is given to the provider in determining who may receive the procedure, as having the procedure deemed "medically necessary" previously enabled sterilization to be provided at the time of Cesarean section when sterilization was illegal [30]. This discretionary decision-making is also likely affected by provider perceptions towards HIV, potentially reducing respect for patient autonomy. As a result, regret associated with a coerced procedure or inability to get a desired procedure due to local practice standards have been noted in prior studies in Brazil, with greater advocacy for rights-based counseling for HIV-positive women [11,31].

A few women reported experiences of stigma and prejudice and did not feel as if their questions and concerns were received without judgment or that their providers and other health professionals were interested in or prepared to give them advice 
on reproductive health. In addition to the patients feeling judged in several instances by their providers, some patients used selfjudging language at times regarding their struggles to maintain protective sexual behaviors, and some reported difficult experiences related to domestic and/or sexual violence. In addition to accessing accurate information regarding safe sex, deeper psychosocial issues such as internalized stigma and self-acceptance may be impacting both the quality of life and the health-related behaviors of women living with HIV/AIDS and should be contemplated within future intervention research regarding patient-provider communication. Perceived stigma was already identified by our group as a key predictor of poor care-seeking in the field of sexually transmitted infections [32]. The individual and environmental barriers that impede contraceptive use and safe sex, such as fear of domestic violence or the sense that poor quality of care is a deserved punishment for having HIV, indicate a need for psychological and social support from trained professionals. Outside Brazilian reference centers psychology and social work services are usually understaffed; these services are greatly needed and efforts should be made to include them through the public system or through advocacy and support groups.

One way to improve patient-provider communication is to implement continuing education and training for health care providers. Previous studies conducted in Brazil already suggested that health providers working with PLWHA do not feel prepared and/or do not have sufficient time to undergo a thorough discussion about sensitive issues with their patients $[25,26]$. Richardson et al. saw significant reductions in sexual risk behaviors among PLWHA with multiple partners within the context of a safer sex counseling intervention initiated by physicians in the United States [33]. Communication training should ideally shift the focus from provider to client; family planning programming using a client-centered approach resulted in greater method satisfaction and continuation without requiring service structure change in Egypt [34].

We elected to use FGD prior to IDI and FLI to establish the predominant themes for exploration of the study topics. The FGD also served an unanticipated purpose as a group information and support session. We believe the group information session approach may be an efficient means to disseminate reproductive health information without placing undue burden on a trained provider. This approach has been used successfully as workshops among female sec workers in Belo Horizonte, Brazil [35]. However, sufficient training of the provider/moderator in group dynamics and attention to group composition will be needed to maximize benefit from this approach.

The major limitation of this study is the reliance on selfreported data, which is typical for studies addressing sensitive issues such as sexual behavior. The evaluation of beliefs and behaviors related to sensitive topics through self-report might lead to some "socially acceptable" responses, such as consistent condom use, that may have been over-reported. The study site (a medical facility) may have created a degree of deference bias, with the study population responding to questions in order to please the interviewers and facilitators. The small sample size is also a limitation as it is possible, though unlikely, that the findings are not transferrable to other settings or populations. Another limitation is the lack of information on the actual content of the patient-provider interaction or provider perspectives. Future studies should combine both patient and provider interviews with direct observations of routine consultations, similar to that conducted by Fehringer et al. [26].

In order to address those limitations, we used three different methods for data collection: in-depth interviews, focus group discussion, and freelists interviews. The findings were complementary, and we did not identify any conflicts or different point of views according to method of data collection. However, focus groups participants were more likely to discuss general problems and less likely to address personal concerns and experiences. Indepth interview participants addressed mostly issues related to highly sensitive problems (e.g. stigma, domestic violence), while those interviewed using the freelist method (a brief and more objective interview) were more likely to address aspects related to patient-provider communication, Therefore, the vast majority quotes selected for this paper were collected using this method.

\subsection{Conclusions}

Despite these limitations, the data presented herein illuminate areas of patient-provider communication, which, if improved, may lead to safer sexual behavior, better reproductive health, and improved well-being among HIV-positive women. Key areas for improvement identified by this study include lack of time for discussion of reproductive health issues during routine patient visits, provider assumptions regarding appropriate contraceptive use for HIV-positive women, and limited patient knowledge and comfort in initiating reproductive health discussions with providers.

\subsection{Practice implications}

Based on participant feedback during the study, we propose a group information session or support group construct as a means of efficient, acceptable information dissemination in this context. Group moderators would receive extensive training in both reproductive health issues specific to HIV-positive women and in managing group dynamics. Issues raised in a group setting whose sensitivity preclude extensive group discussion (e.g. forced sex, pregnancy termination) may then be raised during provider visits. Both in preparation for this possible intervention format and to address key deficiencies, we further recommend focused training in reproductive health with emphasis on a client-centered experience for HIV-care providers. Last, a greater degree of coordination or integration should be fostered between HIV and reproductive health services to ensure that problems outside the scope of routine HIV care are promptly and correctly referred. Future directions for research include development and testing of the proposed group-based intervention model, both through impact on contraceptive utilization and on participant empowerment to address reproductive health issues with providers.

HIV-care providers need to address a range of reproductive health needs, either by referral or - whenever feasible - by directly providing these services. PLWHA will have continuing reproductive health desires and needs that must be attended to. Many will want to have a baby, whereas others will want to avoid pregnancy. And as more HIV-positive people live longer, healthier lives, this reality will only intensify. The synergies resulting from integrating reproductive health services into HIV-related programs will inevitably accelerate progress towards achieving a better and more comprehensive treatment for PLWHA. As the HIV pandemic evolves, countries can no longer afford to overlook the new opportunities arising from integration.

\section{Conflict of interest}

The authors declare that we have no conflict of interest.

\section{Acknowledgments}

The authors thank the participants for their time and constructive comments. This study was funded by the Morris Smith Foundation, the Doris Duke Charitable Foundation, and the Special Programme of Research, Development and Research 
Training in Human Reproduction, World Health Organization. The authors would also like to recognize the contributions of Dr. Maria I. Linhares and Dr. Louise Bastos Schilkowsky, as well as and the staff at Ambulatório da Providência and Hospital Escola São Francisco de Assis.

\section{References}

[1] Joint United Nations Program on HIV/AIDS (UNAIDS). Report on the global AIDS epidemic. Geneva: UNAIDS; 2008.

[2] Wilcher R, Cates Jr W, Gregson S. Family planning and HIV: strange bedfellows no longer. AIDS 2009;23:S1-6.

[3] Spaulding AB, Brickley DB, Kennedy C, Almers L, Packel L, Mirjahangir J, et al. Linking family planning with HIV/AIDS interventions: a systematic review of the evidence. AIDS 2009;23:S79-88.

[4] Cooper D, Moodley J, Zweigenthal V, Bekker LG, Shah I, Myer L. Fertility intentions and reproductive health care needs of people living with HIV in Cape Town, South Africa: implications for integrating reproductive health and HIV care services. AIDS Behav 2009;38-46.

[5] Nunn AS, da Fonseca EM, Bastos FI, Gruskin S. AIDS treatment in Brazil: impacts and challenges. Health Affair (Millwood) 2009;28:1103-13.

[6] Myer L, Rebe K, Morroni C. Missed opportunities to address reproductive health care needs among HIV-infected women in antiretroviral therapy programmes. Trop Med Int Health 2007;12:1484-9.

[7] Kim YM, Davila C, Tellez C, Kols A. Evaluation of the World Health Organization's family planning decision-making tool: improving health communication in Nicaragua. Patient Educ Couns 2007;66:235-42.

[8] Loh A, Simon D, Wills CE, Kriston L, Niebling W, Härter M. The effects of a shared decision-making intervention in primary care of depression: a clusterrandomized controlled trial. Patient Educ Couns 2007;67:324-32.

[9] Newbold KB, Willinsky J. Providing family planning and reproductive healthcare to Canadian immigrants: perceptions of healthcare providers. Cult Health Sex 2009;11:369-82.

[10] Paiva V, Santos N, França-Junior I, Filipe E, Ayres JR, Segurado A. Desire to have children: gender and reproductive rights of men and women living with HIV: a challenge to health care in Brazil. AIDS Patient Care ST 2007;21:268-77.

[11] Oliveira F, Kerr L, Frota A, Nóbrega A, Bruno Z, Leitão T, et al. HIV-positive women in northeast Brazil: tubal sterilization, medical recommendation and reproductive rights. AIDS Care 2007;19:1258-65.

[12] Segurado AC, Paiva V. Rights of HIV positive people to sexual and reproductive health: parenthood. Reprod Health Matters 2007;15:27-45.

[13] Galvão MT, Cerqueira AT, Marcondes-Machado J. Contraceptive measures and HIV transmission protection among women with HIV/AIDS. Rev Saude Publ 2004;38:194-200.

[14] Santos NJ, Buchalla CM, Fillipe EV, Bugamelli L, Garcia S, Paiva V. Reproduction and sexuality in HIV-positive women, Brazil. Rev Saude Publ 2002;36:12-23.

[15] Paiva V, Latorre Mdo R, Gravato N, Lacerda R, Ayres JR, Segurado A, et al. Enhancing care initiative - Brazil. Sexuality of women living with HIV/AIDS in São Paulo. Cad Saude Publ 2002;18:1609-20.

[16] Bernard HR. Social research methods: qualitative and quantitative approaches. Sage Publications Inc.; 2000
[17] Malta MS, Todd CS, Stibich MA, Garcia T, Pacheco D, Bastos FI. Factors influencing contraceptive choice and discontinuation among HIV-positive women in Rio de Janeiro, Brazil. In: Oral presentation, international conference on family planning: research and best practices; 2009 [Abstract\# 502].

[18] Borgatti SP. Elicitation techniques for cultural domain analysis. In: Schensul JJ, editor. The ethnographer's toolkit Enhanced ethnographic methods audiovisual techniques focused group interviews and elicitation, vol. 3. Thousand Oaks, CA: Sage Publications; 1999. p. 115-51.

[19] Quinlan M. Considerations for collecting freelists in the field: examples from ethobotany. Field Methods 2005;17:219-34.

[20] Weller SC, Romney AK. Systematic data collection. Thousand Oaks, CA: Sage Publications; 1988.

[21] Denzin NK. In: Lincoln Y, editor. The SAGE handbook of qualitative research 3rd Edition, Sage Publications, Inc.; 2005.

[22] Stauss A, Corbin J. Basics of qualitative research: grounded theory procedures and techniques, 2nd Edition, Newbury Park, CA: Sage Publications; 1998.

[23] Borgatti SP. ANTHROPAC 4.0. Natick, MA: Analytic Technologies; 1996.

[24] Smith JJ. Using ANTHROPAC 3.5 and a spreadsheet to compute a freelist salience index. CAM Newslett 1993;5:1-3.

[25] Malta M, Petersen ML, Clair S, Freitas F, Bastos FI. Adherence to antiretroviral therapy: a qualitative study with physicians from Rio de Janeiro, Brazil. Cad Saude Publ 2005;21:1424-32.

[26] Fehringer J, Bastos FI, Massard E, Maia L, Pilotto JH, Kerrigan D. Supporting adherence to highly active antiretroviral therapy and protected sex among people living with HIV/AIDS: the role of patient-provider communication in Rio de Janeiro, Brazil. AIDS Patient Care ST 2006;20:637-48.

[27] Johnson KB, Akwara P, Rutstein SO, Bernstein S. Fertility preferences and the need for contraception among women living with HIV: the basis for a joint action agenda. AIDS 2009;23:S7-17.

[28] Leite IC, Gupta N. Assessing regional differences in contraceptive discontinuation, failure and switching in Brazil. Reprod Health 2007;4:6.

[29] Brazilian Ministry of Health. Demography and health survey of women and child (PNDS 2006). Brasília: Brazilian Ministry of Health; 2009.

[30] Osis MJ, de Souza MH, Faúndes A. Brazil: comparative study of the consequences of tubal ligation for women's lives in Campinas. Brasilia, Brazil: Family Health International; 1998.

[31] Hopkins K, Maria Barbosa R, Riva Knauth D, Potter JE. The impact of health care providers on female sterilization among HIV-positive women in Brazil. Soc Sci Med 2005;61:541-54.

[32] Malta M, Bastos FI, Strathdee SA, Cunnigham SD, Pilotto JH, Kerrigan D. Knowledge, perceived stigma, and care-seeking experiences for sexually transmitted infections: a qualitative study from the perspective of public clinic attendees in Rio de Janeiro, Brazil. BMC Public Health 2007;7:18.

[33] Richardson JL, Milam J, McCutchan A, Stoyanoff S, Bolan R, Weiss J, et al. Effect of brief safer-sex counseling by medical providers to HIV-1 seropositive patients: a multi-clinic assessment. AIDS 2004;18:1179-86.

[34] Abdel-Tawab N, Roter D. The relevance of client-centered communication to family planning settings in developing countries: lessons from the Egyptian experience. Soc Sci Med 2002;54:1357-68.

[35] Chacham AS, Diniz SG, Maia MB, Galati AF, Mirim LA. Sexual and reproductive health needs of sex workers: two feminist projects in Brazil. Reprod Health Matters 2007;15:108-18. 